\title{
METÁSTASIS CUTÁNEA DE CARCINOMA DIFERENCIADO DE TIROIDES. A PROPÓSITO DE UN CASO.
}

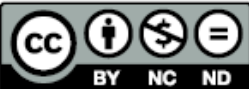
Este artículo está bajo una icencia de Creative Commons de tipo Reconocimien- No comercial - Sin obras OPEN ACCESS derivadas 4.0 International.

1 Hospital de Especialidades Carlos Andrade Marín HECAM. Médico del Servicio de Cirugía Oncológica. Quito - Ecuador

\section{ORCID ID:}

Sánchez Urresta Carlos

orcid.org/0000-0002-7332-9435

Palacios Cortes Christian

orcid.org/0000-0003-2114-7637

Jaramillo Calvas Oscar

orcid.org/0000-0002-9735-2306

*Corresponding author: Sánchez

Urresta Carlos.

E-mail: carlosgs797@gmail.com

Article history

Received: 23 - Apr - 2019

Accepted: 04 - Jul - 2020

Publish: 01 - Aug - 2020

CARE 2017 Check List statement: The author have real the CARE 2017 Check List and the manuscript was prepared and revised according to the CARE 2017 Checklist.

\section{Conflict of interest: All authors declared that} there are no conflicts of interest.

Financial disclosure: The authors have no financial relationships relevant to this article to disclose.

Authors' contribution: All the authors contributed in the search, selection of articles and writing. All the authors reviewed and approved the final manuscript.

Forma de citar este artículo: Sánchez Urresta C, Palácios Cortes C, Jaramillo Calvas O. METÁSTASIS CUTÁNEA DE CARCINOMA DIFERENCIADO DE TIROIDES. A PROPÓSITO DE UN CASO Rev Med Vozandes. 2020; 31 (2): 84 - 89

\section{Resumen}

El cáncer de tiroides (CT) así como otros tipos de tumores malignos tienen la capacidad de invadir e infiltrar otros tejidos tanto contiguos como tejidos y órganos distantes produciendo lo que se conoce como metástasis (MT). Esta es una característica propia de la mayoría de los tumores cancerígenos, y el mecanismo por el cual lo realizan se produce de distintas maneras dependiendo del tipo histológico del tumor, así como de sus particularidades de diseminación propias sea vía linfática, hematógena, nerviosa o por invasión local.

Hablando particularmente del cáncer de tiroides (CT) y siendo más específicos el cáncer papilar (CPT), es la variante histológica maligna que con más prevalencia e incidencia se presenta en esta glándula, además que es el cáncer tiroideo que presenta el mejor pronostico y más alternativas de tratamiento por su patrón de crecimiento lento, y por su baja capacidad de producir metástasis (MT) a distancia, presentado se en menos del $10 \%$ de pacientes con este diagnóstico.

Pese a esta baja tasa de enfermedad metastásica, esta patología ha presentado un crecimiento notable en su prevalencia llegando a triplicarse en los últimos 30 años, lo que ha originado de la misma manera un aumento en la prevalencia de casos de metástasis (MT) por esta neoplasia.

Cuando se presenta enfermedad metastásica del carcinoma papilar de tiroides (CPT) se localiza con mayor frecuencia en tejido ganglionar seguido por tejido pulmonar y óseo. La diseminación de este tipo de cáncer a otros sitios es infrecuente, y a piel es aún más inusual. Por este motivo se presenta el caso de una paciente femenina de 74 años, con antecedentes de diagnóstico de carcinoma diferenciado de tiroides sometida a varias intervenciones quirurgicas previas, la cual presenta una lesión dérmica a nivel pre esternal, cuyo estudio histopatológico post resección fue compatible con diseminación metastásica cutánea de este tipo de cáncer.

Palabras clave: Cáncer de tiroides, Carcinoma papilar de tiroides, Cáncer de piel

\section{Abstract \\ CUTANEOUS METASTASIS OF DIFFERENTIATED THYROID CARCINOMA. ABOUT A CASE.}

Thyroid cancer (CT) as well as other types of malignant tumors have the ability to invade and infiltrate other tissues both contiguous and distant tissues and organs producing what is known as metastasis (MT). This is a characteristic of the majority of cancerous tumors, and the mechanism by which they are carried out occurs in different ways depending on the histological type of the tumor, as well as its own dissemination characteristics, whether lymphatic, hematogenous, nervous or local invasion.

Speaking particularly of thyroid cancer (CT) and being more specific papillary cancer (CPT), it is the malignant histological variant that has the most prevalence and incidence in this gland, in addition it is the thyroid cancer that has the best prognosis and more treatment alternatives due to their slow growth pattern, and due to their low capacity to produce distance metastases (MT) presented in less than $10 \%$ of patients with this diagnosis.

Despite this low rate of metastatic disease, this pathology has presented a remarkable growth in its prevalence, tripling in the last 30 years, which has also caused an increase in the prevalence of metastatic cases (MT) due to this neoplasia.

When there is metastatic disease of papillary thyroid carcinoma (CPT), it is most often located in lymph tissue followed by lung and bone tissue. The spread of this type of cancer to other sites is uncommon, and the skin is even more unusual. For this reason, the case of a 74-year-old female patient is presented, with a history of diagnosis of differentiated thyroid carcinoma undergoing several previous surgical interventions, which presents a dermal lesion at the pre-sternal level, whose post-resection histopathological study was compatible with metastatic skin spread of this type of cancer.

Keywords: Thyroid Cancer, Papillary Thyroid Carcinoma, Skin Cancers 


\section{Introducción}

El cáncer de tiroides ocupa entre el 1 al 2\% de todos los tipos de neoplasias malignas que afectan al ser humano y es la neoplasia de tipo endocrinológico más frecuente, afecta principalmente a mujeres en su quinta década de vida, pero cada vez se está convirtiendo en un diagnostico más frecuente tanto mujeres como hombres y en edades más tempranas ${ }^{1-3}$.

En el Ecuador no dispone de datos epidemiológicos nacionales que muestren el real Burden Epidemiológico de esta patología, sin embargo, la información referente obtenida de la Sociedad de Lucha contra el Cáncer (SOLCA) muestra claramente como existen cambios dramáticos en la epidemiologia del CT en los últimos 15 años. Observándose que tanto en hombres como en mujeres la tasa estándar de incidencia y mortalidad se ha llegado a duplicar, similar con lo que está aconteciendo a nivel global ${ }^{4,5}$.

Además, diversos estudios epidemiológicos realizados en la región de América del Sur, muestran que hay una prevalencia ligeramente más elevada en comparación con países más desarrollados y al igual que en estos, el sexo femenino es el más representativo abarcando casi el $80 \%$ de la población afectada con un pico de incidencia en la adultez entre los 40 a 50 años de edad ${ }^{(6,7)}$

Dentro de las múltiples variantes histológicas, el carcinoma diferenciado y dentro de este subgrupo, el CPT es el cáncer tiroideo más frecuente, teniendo una prevalencia aproximada del $80 \%$ de todos los tumores malignos que afectan esta glándula ${ }^{(8)}$.

La importancia de esta variante histológica radica en que, además de ser la más prevalente el CPT tiene un patrón de crecimiento muy lento, con progresión de enfermedad limitada y además una capacidad metastásica sumamente baja. Sin embargo, cuando esta se presenta, generalmente lo hace invadiendo de manera local y regional los tejidos más adyacentes a la glándula tiroides ${ }^{(1,9)}$. Los casos de progresión tumoral a distancia, hacia otros sitios, tejidos blandos y órganos más distantes son aún más infrecuentes y según reportes de la literatura se presenta en menos del $10 \%$ de personas que han sido diagnosticadas con esta enfermedad maligna $(1,2,8)$.

En los últimos años, se ha reportado un incremento en la incidencia de tumores malignos tiroideos y metástasis originadas por estos, y el Ecuador, no ha sido la excepción, la incidencia se ha incrementado llegando a ser 20 veces más frecuente en comparación con la incidencia reportada en el estudio de Salazar et al. (7). Numerosas causas están siendo investigadas y se han establecido como el origen de este incremento tan abrupto de la incidencia, como son la pesquisa cada vez más temprana de esta patología, la facilidad para acceder a numerosas técnicas diagnósticas como son la ecografía y la punción de aguja fina (PAAF), el mejoramiento y capacitación del personal médico que permite evaluar cada vez nódulos tiroideos más pequeños $(1,2,9,10)$. Con respecto al Ecuador se están tratando de filiar otras razones como posibles causas de esta alta incidencia, como por ejemplo la relación entre el CT con la altitud, razón que explicaría la mayor prevalencia de esta enfermedad en la región andina de nuestro país, y así mismo la relación que existe entre este cáncer y la cercanía con regiones volcánicas como ya ha sido establecido en otros sitios como en Sicilia Italia $(7,11)$, de todas maneras no se cuenta aún con resultados estadísticos en nuestro medio que aseveren estas sospechas.

El aumento de la incidencia global del CT, ha conllevado la implementación de programas de screening, permitiendo así diagnósticos precoces y en etapas tempranas del CT, pese a esto la incidencia de MET a distancia producida por esta patología tumoral sigue siendo relevante (12). Este tipo de MET pueden afectar una gran variedad de órganos, siendo el tejido pulmonar y óseo los más afectados $(1,2,13)$. La afectación de otros órganos y la afectación cutánea es aún más inusual, según reportes de casos esta última varía entre un 3 a $5 \%$ de los casos de MET a distancia. Por lo general se encuentra asociada a una enfermedad activa de muchos años de evolución, mal controlada y con evidencia de enfermedad metastásica a otros sitios de manera simultánea ${ }^{(2,8,14,15)}$.

Debido a la infrecuencia en la presentación de este tipo de metástasis y afectación cutánea, se realiza el reporte del caso a continuación.

El presente estudio sigue las recomendaciones de las directrices CARE (consensus-based clinical case reporting guideline) $)^{16}$ para el reporte de casos, el consentimiento informado para la publicación con fines académicos y guardando toda la confidencialidad posible fue firmado por el paciente.

\section{Presentación del Caso}

Se presenta el caso de una paciente femenina de 72 años, domiciliaria en Cayambe - Ecuador, la cual niega tener antecedentes familiares con diagnóstico oncológico y presenta como antecedente patológico personal haber sido diagnosticada de CPT en el año 2013, motivo por el cual fue sometida a una tiroidectomía total tres meses después del diagnóstico inicial. Posteriormente a esta primera intervención quirúrgica se complementa el tratamiento con la administración de dosis ablativa de yodo radiactivo (100 mCi) y a continuación se mantiene en seguimiento por endocrinología y en tratamiento con supresión de la Hormona liberadora de tirotropina (TSH) mediante administración de Levotiroxina.

En el año 2017, cuatro años después de la haber sido sometida a la tiroidectomía total acude a una nueva consulta quirúrgica por la presencia de una adenopatía cervical identificada en una ecografía de seguimiento, de la cual se realizó una PAAF y se complementó con el respectivo estudio citológico, determinando así la presencia de enfermedad metastásica del CPT a nivel ganglionar cervical lateral derecho. Debido a estos hallazgos es sometida a una segunda intervención quirúrgica en este caso un vaciamiento cervical radical modificado derecho, y se complementa el tratamiento con una nueva dosis ablativa de yodo 131 (100 mCi). 
Posteriormente, en el año 2018 debido a la respuesta bioquímica incompleta y a la evidencia de niveles elevados persistentes de tiroglobulina (Tg), se solicitó un nuevo un rastreo corporal. Después de la administración de Yodo 131 (5 mCi), se logró identificar lesiones hiper captantes compatibles con tejido tiroideo metastásico a nivel pulmonar y hepático, y al examen físico se logró observar una lesión de tipo nodular, de consistencia blanda, móvil, regular, no dolorosa, ubicada a nivel de la cara anterior del cuerpo del esternón, iodo refractaria según el resultado del rastreo corporal (Figura 1).

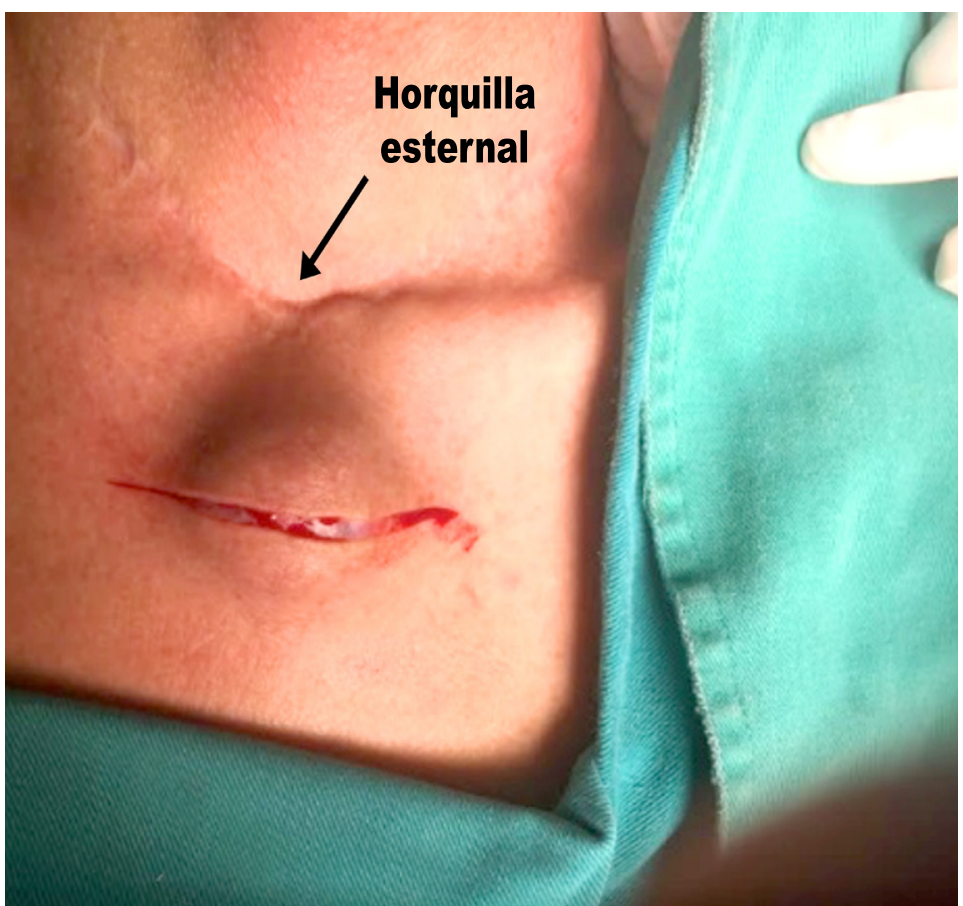

Figura 1 . Lesión a nivel pre esternal por debajo de la horquilla esternal

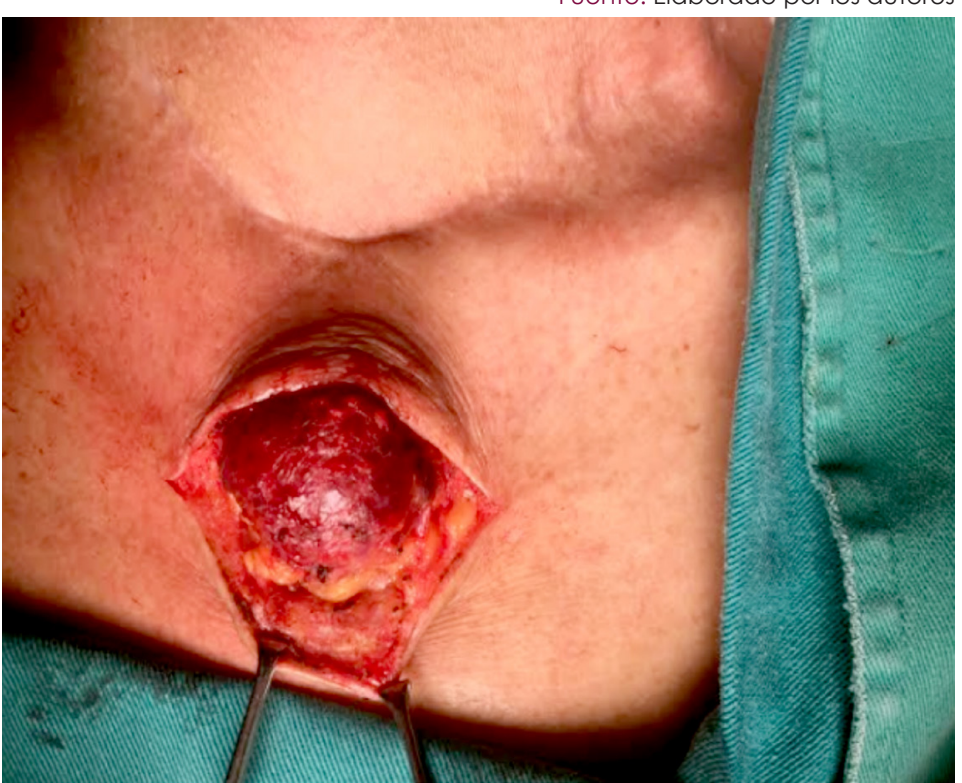

Figura 2. Procedimiento quirúrgico, formación de flaps sub dérmicos para disección del tumor.

Fuente: Elaborado por los autores
Ante estos hallazgos tanto en estudios de imagen como al examen físico se plantea la sospecha clínica de enfermedad MET cutánea del CT y con el fin de descartar alguna otra enfermedad maligna a este nivel se solicita una biopsia dirigida por ecografía de dicha lesión dérmica. El resultado del estudio citológico fue reportado como metástasis de CPT.

Con este resultado se decide realizar la resección quirúrgica de la tumoración pre-esternal, la cual se presenta macroscópicamente como un tumor blando, violáceo, ubicado a nivel subdérmico, sin erosión o infiltración hacia tejido óseo adyacente, de contornos regulares, que mide aproximadamente $3 \times 2,5 \mathrm{~cm}$. en sus mayores diámetros, de superficie lisa. La pieza operatoria es extraída y enviada para estudio anatomopatológico. (Figura 2 y 3).

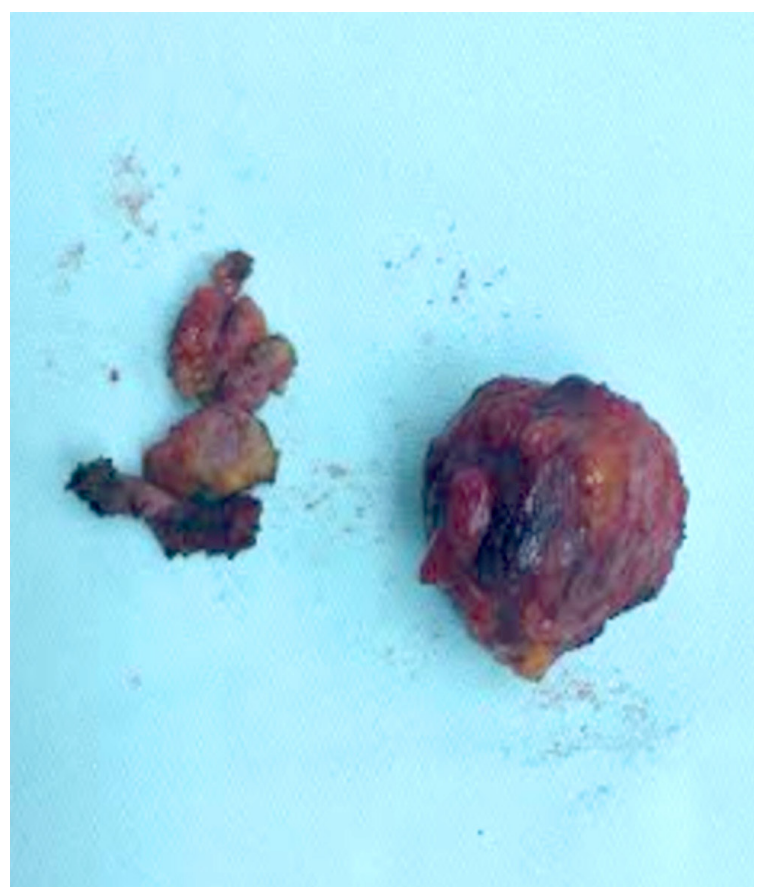

Figura 3 . Pieza quirúrgica, lesión pre esternal.

Fuente: Elaborado por los autores

El análisis anatomopatológico de la masa extraída demostró metástasis de carcinoma papilar de tiroides moderadamente diferenciado con patrón folicular en el $80 \%$, cuyos bordes de resección son libres de enfermedad residual (Figura 4 y 5).

La paciente permaneció hospitalizada por 24 horas y fue dada de alta en buenas condiciones generales. Se realizaron controles médicos por consulta externa en el pos operatorio mediato y tardío, con procesos cicatrízales adecuados y una evolución pos quirúrgica satisfactoria. Actualmente se mantiene en controles periódicos semestrales, recibe tratamiento supresor con Levotiroxina por sus lesiones metastásicas hepáticas y pulmonares las cuales no son tratables quirúrgicamente, no se ha encontrado nuevas lesiones dérmicas. 


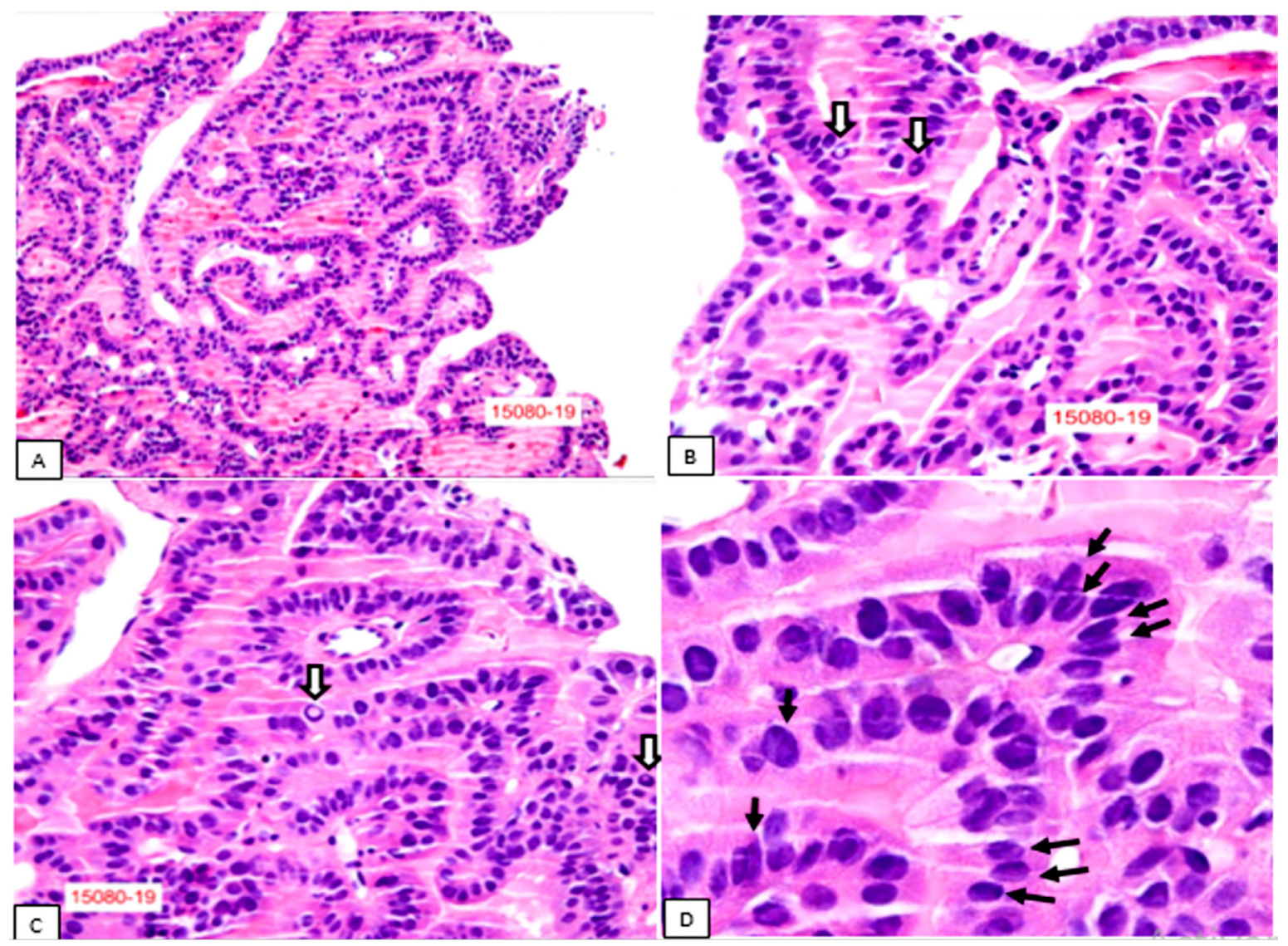

Figura 4. Lesión tumoral compuesta por folículos (A) que con mayor aumento presentan revestimiento epitelial en una sola hilera de células cúbicas y columnares bajas (B), algunas inclusiones intranucleares (B y C) y hendiduras intranucleares (D).

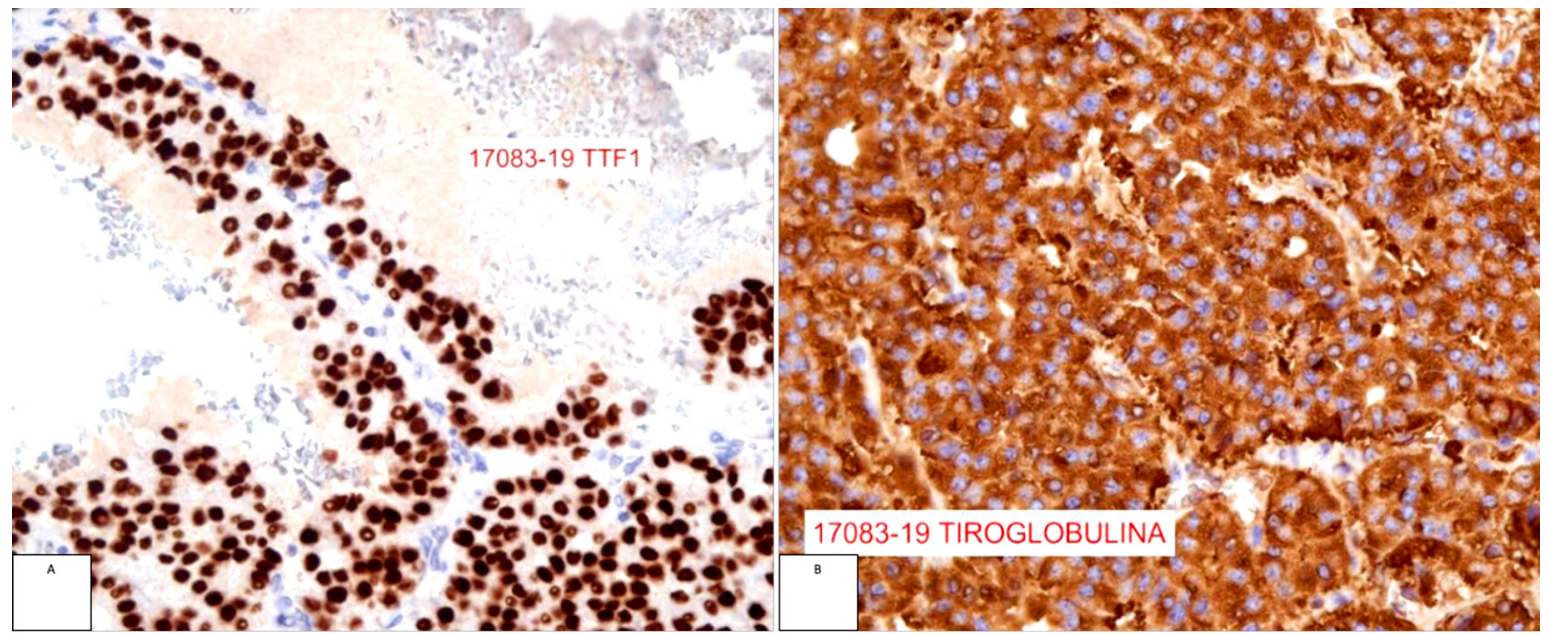

Figura 5. Estudio inmunhistoquímico del tumor pre esternal. (A): tinción TTF-1 (Factor 1 de Transcripción Tiroidea) se observa la expresión difusa del ARNm a nivel del epitelio tiroideo característica del Cáncer papilar de tiroides. (B) tinción con tiroglobulina y captación del mismo de manera difusa orientando hacia el origen tiroideo de la muestra estudiada. 


\section{Discusión}

El CPT es una neoplasia maligna con una tendencia de crecimiento y progresión de enfermedad generalmente paulatino y lento en comparación con otro tipo de tumores malignos incluidas otras variantes histológicas que afectan a esta glándula ${ }^{(13)}$. Este patrón de diseminación hace que el hallazgo de extensión de esta enfermedad hacia otros órganos y tejidos blandos distantes represente apenas un subgrupo muy reducido de pacientes. Y así mismo, en caso de presentarse los tejidos y órganos principalmente afectados son aquellos con íntima relación con el parénquima glandular tiroideo; como son el tejido ganglionar loco regional del cuello en primer lugar por ser el sitio de drenaje linfático próximo a la tiroides, y el parénquima pulmonar igualmente afectado por la proximidad con la vía linfática del cuello ${ }^{(9)}$. Las metástasis hacia otros órganos y tejidos son hallazgos más peculiares, afectando a órganos como: cerebro, hígado y piel entre el 3 al $5 \%$ de pacientes con enfermedad a distancia $(1,13)$.

Aproximadamente la mitad de los casos que presentan MT de este tipo de cáncer son identificados al momento del diagnóstico, el resto de los casos son identificados durante el seguimiento posterior en particular el realizado durante el primer año, y pudiendo incluso identificarse enfermedad metastásica posterior a los 10 años de seguimiento hasta en un $15 \%$ de pacientes ${ }^{(1)}$.

La mayoría de los casos que presentan MT del cáncer papilar de tiroides son asintomáticos (17), y se presentan por lo general en aquellos pacientes con tumores grandes, voluminosos y con una invasión y extensión local importante con gran infiltración tanto vascular como linfática lo que facilita la diseminación a distancia $(12,13,17)$.

La guía clínica de Nódulo Tiroideo y Cáncer Diferenciado de Tiroides publicada en el Ecuador en 2019, refiere que el $4 \%$ al $15 \%$ de los pacientes con CT realizan MT a distancia, con una sobrevida del $50 \%$ a 10 años, las metástasis a tejidos blandos son consideradas inusuales (11).

Debido a este bajo nivel de incidencia de metástasis de carcinoma papilar de tiroides hacia piel, no se ha logrado establecer ni describir características específicas de este tipo de lesiones cutáneas, pero en la mayoría de casos dichas lesiones se presentan como un lesión dérmica de tipo placa o nódulo eritemato-violáceo, blando, regular, no infiltrante, pruriginosa, hiper vascularizada y que afectan sobre todo áreas dérmicas de alto aporte sanguíneo en especial de la cabeza y el cuello $(2,8,14,15)$.

En pacientes con diagnóstico previo de cáncer de tiroides, con enfermedad sistémica conocida, y que presenten lesiones cutáneas con las características previamente descritas, se debe tener una alta sospecha clínica de cáncer metastásico de tiroides a este nivel, no obstante, los pacientes deben ser sometidos a una biopsia de dichas lesiones cutáneas para asegurar el origen tiroideo ya que pueden ser confundidas con otros tumores malignos propios de la piel como son el carcinoma baso celular o el carcinoma escamo celular ${ }^{(15,18)}$.

Las recomendaciones de la ATA 2015, refieren que, en caso de metástasis de carcinoma de tiroides que son iodo - refractarias como es el caso de nuestra paciente, y al no implicar una sintomatología que afecte a su desempeño, el tratamiento está enfocado a la resección del tumor siempre que sea posible. Igualmente se recomienda estudios de imagen que identifiquen otros posibles focos de MT que no hayan sido identificados inicialmente, principalmente a huesos (19).

Con lo que respecta al panorama de manejo de las metástasis a órganos diana, se debe hacer estudios de imagen y seguimiento cada 3 a 12 meses esperando que no exista un crecimiento mayor al $20 \%$, y con lo que respecta a afectación a otros tejidos blandos como la piel, las guías recomiendan la resección o reducción quirúrgica del tumor siempre que sea posible ya que como se conoce, estos sitios anatómicos tienden a ser iodo - refractarios con poca penetración al tratamiento ablativo con $1131^{(9)}$.

\section{Conclusión}

El cáncer diferenciado de tiroides y específicamente el CPT es una neoplasia con una probabilidad metastásica baja. Son escasos los casos descritos por la literatura que se refieren al compromiso dérmico por este tipo de cáncer, por lo cual ante una lesión sospechosa se debe determinar primeramente el origen histológico de la lesión y realizar una resección quirúrgica de la misma siempre que sea posible y en caso de confirmarse el origen tiroideo.

El pronóstico y la sobrevida de esta paciente no varía a la descrita por la literatura y es similar a la establecida en individuos con enfermedad metastásica a distancia hacia otros órganos.

\section{Referencias}

1. Schmidt A, Cross G, Pitoia F. Metástasis a distancia en cáncer diferenciado de tiroides: diagnóstico y tratamiento. Revista Argentina de Endocrinología y Metabolismo. 2017;54(2):92-100.

2. Junquera $S$, Oria E, Tuñón T, Vázquez C, Botella J. Metástasis en cuero cabelludo como primera manifestación de cáncer de tiroides mixto folicular-papilar. 2014. An. Sist. Sanit. Navar. 2014; 37 (2): 281-86.

3. Quinn T, Duncan L, Zembowicz A, Faquin W. Cutaneous Metastases of Follicular
Thyroid Carcinoma. Am J Dermatopathol. 2005;27(4):306-12.

4. Tasas según grupos de edad, por tipo de cáncer. Estadisticas.med.ec. 2020. Available from: http://www.estadisticas.med.ec/webpages/reportes/Graficol-2.jsp

Tasas según grupos de edad, por tipo de cáncer. Estadisticas.med.ec. Available at: http://www.estadisticas.med.ec/webpages/ reportes/Graficol-2.jsp

6. Salazar J, Ortiz E, Solis P, Gómez L, Simbaña K,
Henriquez A, et all. Thyroid Cancer in Ecuador, a 16 years population-based analysis (2001-2016). BMC cancer. 2019;19(1): 294.

Russo M, Malandrino P, Addario W, Dardanoni $G$, Vigneri P, Pellegriti $G$, et all. Several soni $G$, Vigneri P, Pellegriti $G$, et all. Several volcas volcanic area in Sicily. Anticancer research. 2015;35(7): 3995-4001.

Alwaheeb S, Ghazarian D, Boerner SL, Asa SL. Cutaneous manifestations of thyroid cancer: a report of four cases and review of the literature. J Clin Pathol. 2004;57(4):435-8. 
9. Farina E, Monari F, Tallini G, Repaci A, Mazzarotto R, Giunchi F, et al. Unusual Thyroid Carcinoma Metastases: a Case Series and Literature Review. Endocrine Pathology. 2015;27(1):55-64.

10. Corral F, Cueva P, Yépez J, Tarupi W. Trends in cancer incidence and mortality over three decades in Quito-Ecuador. Colombia Médica. $2018 ; 49(1): 35-41$.

11. Nódulo tiroideo y cáncer diferenciado de Tiroides: Diagnóstico, tratamiento y seguimienroides: Diagnostico, tratamiento y seguim
to. Guía Práctica Clínica-MSP. 2019;(1).

12. Faure E, González A, Schwarzstein D, Lutfi R, Puig M. Microcarcinoma papilar de tiroides diagnosticado por una metástasis quistificada en el mediastino. Endocrinología y Nutrición. 2013;60(7):414-16.
13. Høie J, Stenwig A, Kullmann G, Lindegaard M. Distant metastases in papillary thyroid cancer.A review of 91 patients. Cancer $1988 ; 61(1): 1-6$.

14. Márquez A, Ferrándiz L, Ríos J, Camacho F. Metástasis cutáneas craneocervicales de un carcinoma papilar de tiroides variedad folicular. Actas Dermo-Sifiliográficas. 2016;107(1):83-85

15. Lissak B, Vannetzel J, Gallouedec N, Berrod J, Rieu M. Solitary skin metastasis as the presenting feature of differentiated thyroid micro-
carcinoma: Report of two cases. J Endocrino carcinoma: Report of two
Invest. 1995; 18(10):813-16.

16. Gagnier J, Kienle G, Altman D, Moher D, Sox $H$, Riley D. The CARE guidelines: consensusbased clinical case reporting guideline de- velopment. Journal of Medical Case Reports. 2013;7(1).

17. Chisthi M Manju P Thomas P. Carcinoma papilar de tiroides: Úlcera sangrante de piel. Rev Chil Cir. 2015; 67: 643-46.

18. Makris A, Goepel J. Cutaneous metastases from a papillary thyroid carcinoma. British Journal of Dermatology. 1996;135(5):860-61.

19. Haugen B, Alexander E, Bible K, Doherty G, Mandel S, Nikiforov $Y$, et all. American Thyroid Association Management Guidelines for Adult Patients with Thyroid Nodules and DiAdult Patients with Thyroid Nodules and Di-
fferentiated Thyroid Cancer: The American fferentiated Thyroid Cancer: The American
Thyroid Association Guidelines Task Force on Thyroid Nodules and Differentiated Thyroid Cancer. Thyroid. 2016;26(1):1-133. 